\title{
Microsaccadic Suppression of Visual Bursts in the Primate Superior Colliculus
}

\author{
Ziad M. Hafed and Richard J. Krauzlis \\ Systems Neurobiology Laboratory, Salk Institute for Biological Studies, La Jolla, California 92037
}

Saccadic suppression, a behavioral phenomenon in which perceptual thresholds are elevated before, during, and after saccadic eye movements, is an important mechanism for maintaining perceptual stability. However, even during fixation, the eyes never remain still, but undergo movements including microsaccades, drift, and tremor. The neural mechanisms for mediating perceptual stability in the face of these "fixational" movements are not fully understood. Here, we investigated one component of such mechanisms: a neural correlate of microsaccadic suppression. We measured the size of short-latency, stimulus-induced visual bursts in superior colliculus neurons of adult, male rhesus macaques. We found that microsaccades caused $\sim 30 \%$ suppression of the bursts. Suppression started $\sim 70$ $\mathrm{ms}$ before microsaccade onset and ended $\sim 70 \mathrm{~ms}$ after microsaccade end, a time course similar to behavioral measures of this phenomenon in humans. We also identified a new behavioral effect of microsaccadic suppression on saccadic reaction times, even for continuously presented, suprathreshold visual stimuli. These results provide evidence that the superior colliculus is part of the mechanism for suppressing self-generated visual signals during microsaccades that might otherwise disrupt perceptual stability.

\section{Introduction}

Microsaccades are tiny saccades that take place during fixation. These movements can have significant influences on perception. For example, microsaccades can help prevent perceptual fading through jittering retinal images and "refreshing" the activity of sensory neurons in the brain (Martinez-Conde et al., 2004). However, such selfinduced retinal image motion also places constraints on the visual system: to ensure perceptual stability, suppression of visual processing occurs around microsaccades (Zuber and Stark, 1966; Beeler, 1967), similar to that for larger voluntary saccades (Zuber and Stark, 1966; Diamond et al., 2000; Ross et al., 2001; Wurtz, 2008). Understanding the neural origins of microsaccadic suppression is therefore important for uncovering the brain mechanisms of perceptual stability and for understanding the full extent of interactions between microsaccades and vision.

The superior colliculus (SC) likely plays a role in microsaccadic suppression. Sensory responses in this structure are implicated in visual perception and attention (Wurtz and Goldberg, 1972; Wurtz and Mohler, 1976; Fecteau et al., 2004; Boehnke and Munoz, 2008). Moreover, visual bursts in the superficial SC layers are suppressed when the stimuli inducing them appear immediately following voluntary saccades (Robinson and Wurtz, 1976). More recently, studies of SC circuitry in slice preparations have identified possible pathways for mediating saccadic suppression (Lee et al., 2007; Isa and Hall, 2009). The mechanisms for microsaccadic suppression, however, have not been identified.

Received March 5, 2010; revised April 28, 2010; accepted June 8, 2010.

This research was funded by the National Institutes of Health (Grant EY12212).

Correspondence should be addressed to Ziad M. Hafed at his present address: Werner Reichardt Centre for Integrative Neuroscience, Paul Ehrlich Strasse 17, Tuebingen 72076, Germany. E-mail: ziad.m.hafed@cin. uni-tuebingen.de.

DOI:10.1523/JNEUROSCI.1137-10.2010

Copyright $\odot 2010$ the authors $\quad 0270-6474 / 10 / 309542-06 \$ 15.00 / 0$
Here we report that the visual responses of SC neurons are suppressed by microsaccades and identify a behavioral correlate of this suppression in increased saccadic reaction times. Our results show that microsaccades are similar in their influence on sensory processing to larger saccades.

\section{Materials and Methods}

\section{Laboratory setup and animal preparation}

The laboratory setup was identical to that of Hafed and Krauzlis (2008). We collected neuronal data from two ( $\mathrm{W}$ and $\mathrm{J}$ ) adult rhesus monkeys (Macaca mulatta) and behavioral data from monkey W and a third monkey (A). Monkeys were prepared using standard surgical techniques (Hafed and Krauzlis, 2008). All experimental protocols were approved by the Institutional Animal Care and Use Committee and complied with United States Public Health Service policy on the humane care and use of laboratory animals.

\section{Procedures}

Reaction time task. After $230 \mathrm{~ms}$ of fixation of a central spot, the spot jumped to a peripheral location that varied from trial to trial (generally up to $18^{\circ}$ ). Monkeys were instructed to generate a saccade to the new spot location as soon as it appeared. We collected 1214 trials from monkey W (in 19 behavioral sessions) and 1463 from monkey A (in 23 sessions). We did not record neural activity during this task.

Visual stimulus task. Monkeys fixated a small white spot presented at the center of the display over a uniform gray background (Hafed et al., 2009). After $230 \mathrm{~ms}$, a white bar $\left(1.8-6^{\circ}\right.$ high and $0.3-1^{\circ}$ wide $)$ appeared in the periphery and remained on for $750 \mathrm{~ms}$. The bar was oriented perpendicular to the axis connecting its center to the display center, and its dimensions were constant for each recording session [but scaled depending on the SC site as by Hafed and Krauzlis (2008)]. Monkeys were required to maintain fixation (within $1^{\circ}$ ) throughout the trial.

In each session, we presented the bar at up to 11 locations covering the extent of the response field (RF). Five locations were along the axis connecting the display center to the center of the neuron's RF as assessed from an RF mapping task (see below). The bar could appear at the esti- 
A

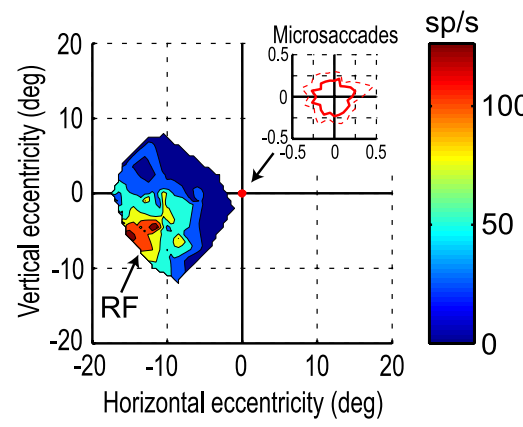

C

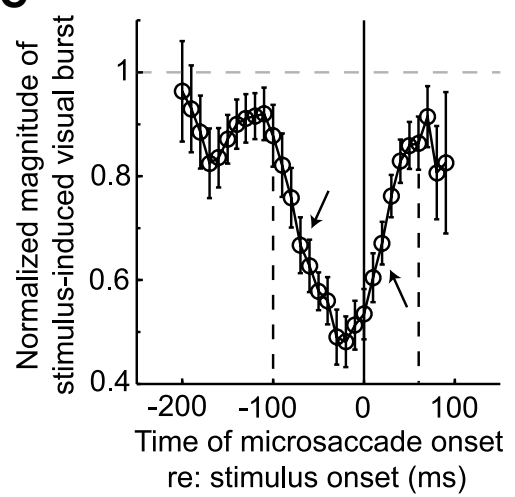

B

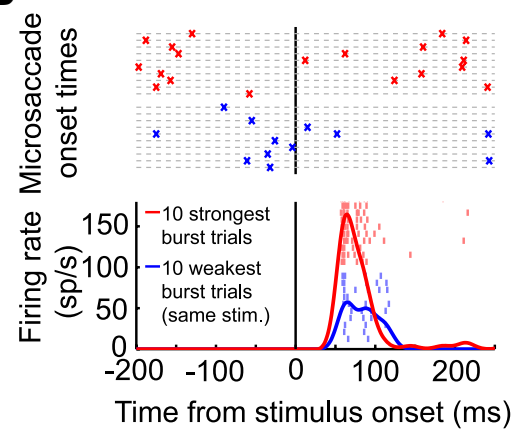

D

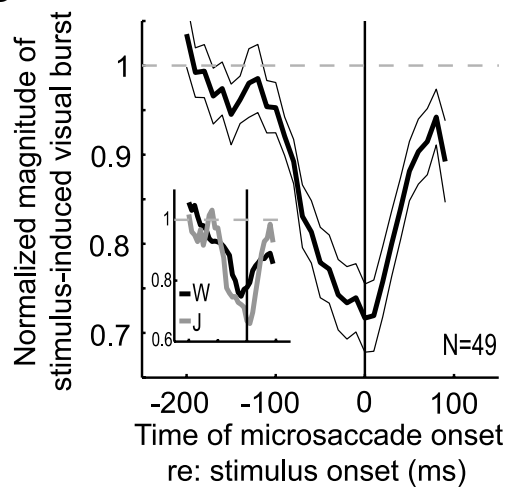

Data analysis

Eye movement analysis. Saccades (and microsaccades) were detected using velocity and acceleration thresholds (Hafed et al., 2009). We first identified peaks in radial eye velocity above a threshold (typically $7 \%$ for microsaccades). Then, regions flanking each peak were flagged if eye velocity remained higher than the velocity threshold. The start and end points of the movements were refined by finding the time points at which eye acceleration in the direction of movement crossed a second threshold (typically $550^{\circ} / \mathrm{s}^{2}$ for microsaccades).

Several measures ensured that detected movements were not simply noise (see supplemental Fig. S1, available at www.jneurosci.org as supplemental material). First, we visually inspected all trials and overruled the automatic detection when misses or false positives were obvious. Second, we plotted the main sequence relationship between amplitude and peak eye velocity for each monkey and related microsaccades to larger saccades, confirming earlier observations (Zuber and Stark, 1965) (supplemental Fig. S1B, available at www.jneurosci.org as supplemental material). We also measured the amplitude distributions of microsaccades (supplemental Fig. $\mathrm{S} 1 C$, available at www.jneurosci.org as supplemental material), corroborating our earlier results (Hafed et al., 2009). Finally, we plotted the time course of microsaccades before and after stimulus onset (supplemental Fig. S1 D, available at www.jneurosci.org as supplemental material) and confirmed a robust reduction in microsaccade rate after stimulus onset followed by a rebound (Rolfs et al., 2008).

We excluded the (few) trials in which there were movements larger than $30^{\prime}\left(0.5^{\circ}\right)$ occurring between -175 and $+150 \mathrm{~ms}$ from stimulus onset. This was done to ensure that we studied the influence of only the smallest eye movements on visual burst suppression.

mated RF center or at $2^{\circ}$ or $4^{\circ}$ farther or nearer eccentricities. Two additional locations were at the RF center but offset by $1^{\circ}$ clockwise or counterclockwise relative to display center. The remaining locations were at the $2^{\circ}$ or $4^{\circ}$ locations farther or nearer than the RF center but offset by $2^{\circ}$ either clockwise or counterclockwise. We collected $25-40$ stimulus repetitions for each bar location.

\section{Single-neuron recordings and neuron classification}

We recorded single-neuron activity from the intermediate SC (1.53 \pm $0.41 \mathrm{~mm}$ SD below surface, $N=49$ neurons -22 in W and 27 in J) during the visual stimulus task. We studied neurons that exhibited activity during a visual interval (50-150 ms after stimulus onset) that was higher ( $p<0.05$, two-tailed $t$ test) than baseline activity ( -50 to $0 \mathrm{~ms}$ relative to stimulus onset) for at least one of our tested bar locations. We also tested neurons for frank saccade-related activity: all were more active in a prelude interval before memory-guided saccades to targets presented near the center of their RFs (75 ms interval starting 100 ms before saccade onset) than during an earlier fixation interval (100 ms before fixation target offset).

\section{RF mapping}

We mapped RFs using a delayed saccade task. For each mapped location, we computed the average firing rate $50-150 \mathrm{~ms}$ after stimulus onset to obtain the visual RF (e.g., Fig. $1 A$ ). To obtain the movement-related RFs (e.g., supplemental Fig. S2A, available at www.jneurosci.org as supplemental material), we computed the peak firing rate during each saccade. Our neurons had preferred eccentricities more than $\sim 6^{\circ}$, resulting in stimulus locations of $4-17^{\circ}$ (mean: $10.3 \pm 3.2 \mathrm{SD}$ ).
Time course of microsaccadic suppression. We used moving averages of $50 \mathrm{~ms}$ bin widths based on the time of microsaccade (onset or end) relative to stimulus onset (or vice versa). Bin centers occurred every 10 $\mathrm{ms}$, and the range of bins was constrained by the initial prescribed fixation interval before stimulus onset $(230 \mathrm{~ms})$ on one end and by "microsaccadic inhibition" after stimulus onset (Rolfs et al., 2008) on the other. We estimated the onset and end of neural suppression effects as the first and last time bins around the movement (or the stimulus onset) for which the $95 \%$ confidence interval of the average activity did not encompass the microsaccade-free baseline activity (described below, Normalization of firing rates). The same procedure was used for the neural and behavioral data, and is modeled after previous behavioral analyses of microsaccadic suppression (Zuber and Stark, 1966; Beeler, 1967). To explore the effect of microsaccade direction relative to stimulus location, we identified the subset of trials that contained microsaccades within $45^{\circ}$ (in direction) of the stimulus location and a second group that contained microsaccades within $45^{\circ}$ of the diametrically opposite location. We statistically compared suppression strength for these two groups of movements by measuring visual bursts when the movements occurred within $30 \mathrm{~ms}$ of stimulus onset (i.e., when maximum suppression was expected).

Normalization of firing rates. Because microsaccades did not occur in every trial, we combined data across trials to obtain sufficient numbers for temporal evolution curves. Because different bar locations presented within a single RF may have themselves altered the visual burst size from trial to trial, we first normalized each neuron's activity for a given stimulus location to a saccade-free measurement. For each bar location, we picked all trials in which there were no microsaccades between -175 and 
$150 \mathrm{~ms}$ from bar onset; we then measured the average firing rate of the neuron during the visual interval $(50-150 \mathrm{~ms})$. This measurement served as the normalization factor, allowing us to investigate microsaccadic suppression of visual bursts across all trials relative to the bursts observed for identical visual stimuli but with no microsaccades.

\section{Results}

Microsaccadic suppression in the superior colliculus

Neurons in the intermediate SC exhibited vigorous visual bursts, as shown by the sample neuron in Figure $1 A$ from the right SC of monkey W. This neuron's visual RF showed a peak in responsiveness at $\sim 16^{\circ}$, with an inner border at $\sim 5^{\circ}$, allowing us to run the visual stimulus task with large stimuli presented within the RF but significantly more eccentric than the eye excursions caused by microsaccades (Fig. $1 A$, red blip at origin; magnified in the inset with the red curve showing mean amplitude at each of 24 equally spaced angular bins, and the dashed line showing the mean + SD contour).

Microsaccades occurring around stimulus onset reduced the amplitude of visual bursts, despite these movements' minute size. An illustration of this phenomenon is shown in Figure $1 B$ for the same neuron as in Figure $1 A$. Using the stimulus location eliciting the highest average response, we sorted individual trials by burst strength. Of the 10 trials with the weakest evoked visual bursts (blue, bottom), nine had microsaccades within $100 \mathrm{~ms}$ from stimulus onset (blue, top). Of the 10 trials with the strongest visual bursts (red, bottom), only three had microsaccades within $100 \mathrm{~ms}$ from stimulus onset (red, top). Thus, this neuron exhibited a correlate of microsaccadic suppression in the size of its visual bursts.

The time course of suppression is reminiscent of classic behavioral suppression phenomena (Zuber and Stark, 1966; Beeler, 1967; Diamond et al., 2000; Ross et al., 2001; Wurtz, 2008). We plotted the magnitude of the same neuron's stimulus-induced visual burst as a function of the time of microsaccade onset relative to stimulus onset (Fig. 1C). Microsaccades starting 10-20 ms before stimulus onset suppressed this neuron's subsequent stimulus-induced visual bursts by $\sim 50 \%$ (Fig. 1C). Moreover, the suppressive effect was present even when the microsaccade preceded stimulus onset by as much as $\sim 100 \mathrm{~ms}$ (indicated by left arrow in Fig. 1C). The suppressive effect also occurred when the stimulus onset occurred slightly before the start of the microsaccades (by up to $\sim 60 \mathrm{~ms}$ ) (indicated by right arrow in Fig. 1C). Thus, the neuron's activity displayed a specific temporal pattern of suppression associated with microsaccades.

The microsaccade-related suppression of visual bursts in the neuron of Figure 1 appears to involve an interaction between the neuron's visual response gain and the occurrence of microsaccades, rather than a direct motor or retinotopic effect. First, the neuron did not exhibit direct microsaccade-related modulations in its activity (supplemental Fig. S2, available at www.jneurosci.org as supplemental material). Second, the microsaccades associated with visual burst

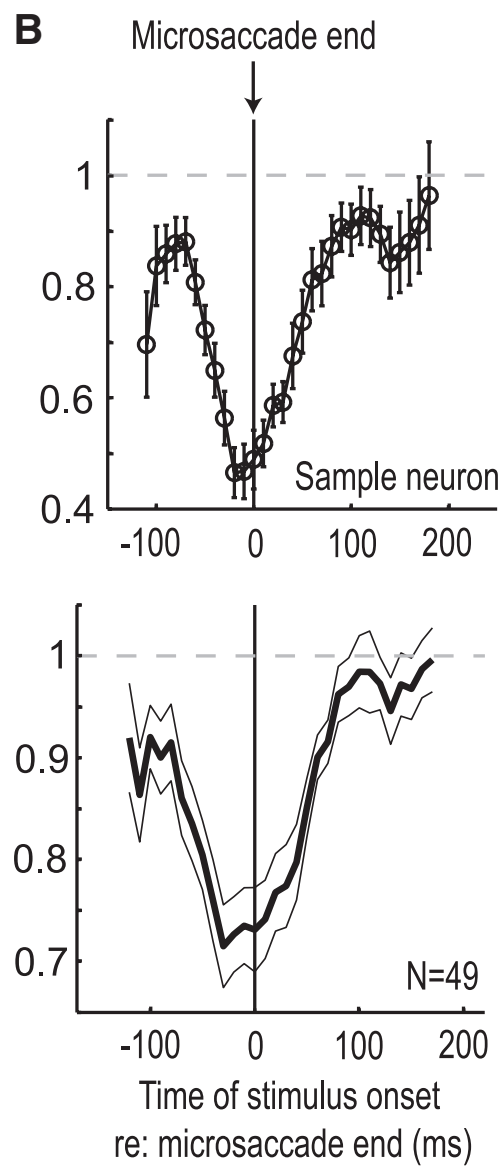

Figure 2. Suppression precedes movement onset and outlasts movement end. $\boldsymbol{A}$, Visual burst suppression data, plotted as a and the neuronal population (bottom). Suppression preceded microsaccade onset by $\sim 70 \mathrm{~ms}$. $\boldsymbol{B}$, Same data aligned on microsaccade end. Suppression outlasted movement end by $\sim 70 \mathrm{~ms}$.

suppression in this neuron were 1-2 orders of magnitude smaller than the size of the neuron's visual RF (Fig. $1 A$, inset), and were also smaller than the extended visual stimuli inducing the bursts. Thus, these movements were too small to displace the stimuli outside the $\mathrm{RF}$, and (because of the spatially extended stimuli) any stimulus displacements caused by microsaccades had large areas of overlap in RF location before and after movement. More importantly, microsaccades occurred in all directions (Fig. $1 A$, inset), ruling out the possibility that microsaccades consistently moved the visual stimuli from preferred to less preferred locations. In fact, dividing movements as being directed either toward or opposite the RF stimulus locations revealed similar suppression ( $p=0.5$, two-tailed $t$ test; $p=0.46$ across neurons). Finally, the suppression could occur even before a microsaccade began (Fig. 1C), suggesting a likely extraretinal origin.

Microsaccadic suppression of visual bursts in the SC occurred consistently across the population of visual-movement neurons we studied in two monkeys (W and J) (Fig. 1D). As in Figure $1 A-C$, these neurons exhibited significant stimulus-induced visual bursts for eccentric stimuli, located at $4-17^{\circ}$. The microsaccades that influenced these neurons' visual bursts had a median amplitude of $\sim 11.5^{\prime}$ and fell short of the proximal edge of the visual and movement-related RFs. Peak visual burst suppression was observed on average across the population when microsaccades occurred at or near stimulus onset (Fig. 1D); this peak suppression had a value of $\sim 30 \%$ when averaged across the neurons. 
To clarify the timing of microsaccade-related suppression, we plotted the strength of stimulus-induced neuronal activity in our population, but now as a function of the time of stimulus onset relative to either movement onset or movement end (Fig. 2). When plotted relative to movement onset, the data (in Fig. $2 \mathrm{~A}$ ) are the same as in Figure 1, $C$ and $D$, but with a flipped time axis. However, this analysis presents the data in a format that is identical to behavioral measures of microsaccadic and saccadic suppression, and demonstrates that stimuli appearing before movement onset could still elicit suppressed visual bursts, even though the movement was yet to occur (Fig. $2 \mathrm{~A}$; negative time values). Moreover, the time course of the suppression across the population (Fig. $2 \mathrm{~A}$, bottom; -70 to $+90 \mathrm{~ms}$ relative to movement onset) shows that suppression could occur even if stimuli appeared only in the wake of microsaccades: suppression persisted for up to $\sim 90 \mathrm{~ms}$ after microsaccade onset, but the microsaccades themselves lasted only $23 \pm 6.5 \mathrm{~ms}$ SD. Thus, microsaccadic suppression of visual bursts preceded movement onset by $\sim 70 \mathrm{~ms}$ (Fig. $2 \mathrm{~A}$ ) and outlasted movement end by $\sim 70$ ms (Fig. 2B).

\section{Behavioral correlate of microsaccadic suppression in reaction times}

Our neural results in the SC suggest that microsaccadic suppression might also affect behavior by increasing saccadic reaction times (RTs), even for stimuli that are high in contrast and continuously present. Consistent with this prediction, microsaccades occurring around stimulus onset caused an increase in saccadic RTs. In an analysis identical to the neuronal one in Figure $2 \mathrm{~A}$ but on the RT task data, we plotted RT as a function of when the peripheral stimulus appeared relative to a microsaccade (Fig. 3). We found an inverted-U-shaped curve, with RTs slower when stimulus and microsaccade onsets were closely synchronized and faster when they were misaligned. This inverted-U-shaped time course resembled the SC neural suppression effects. In fact, for monkey $\mathrm{W}$, for which we had collected neural data as well, we found a strong correspondence between the time course of neural suppression effects in the population of SC neurons and the time course of RT lengthening (Fig. 3A) $(r=-0.903, p<0.0001)$.

These RT influences call for a reinterpretation of previous findings on RT lengthening by microsaccades (Rolfs et al., 2006; Bosman et al., 2009; Kliegl et al., 2009), in which the lengthening was attributed to a motor conflict not a sensory suppression (see supplemental Fig. S3, available at www.jneurosci.org as supplemental material).

\section{Discussion}

We found that stimuli appearing in close temporal register with microsaccades are less effective in eliciting visual bursts in the SC than identical stimuli appearing in the absence of microsaccades. We also found that the time course of such visual burst suppression precedes movement onset and outlasts movement end, consistent with the behavioral correlate of this phenomenon in our RT task. Coupled with previously described saccadic suppression phenomena (Zuber and Stark, 1966; Diamond et al., 2000; Ross et al., 2001; Wurtz, 2008), these results suggest that microsaccades and larger saccades have similar interactions with sensory processing.

The time course of microsaccadic suppression of visual bursts in the SC is similar to the time course of elevated perceptual detection thresholds observed in humans. Zuber and Stark (1966) probed detection performance for brief luminance flashes presented around microsaccades, and found maximal impair-
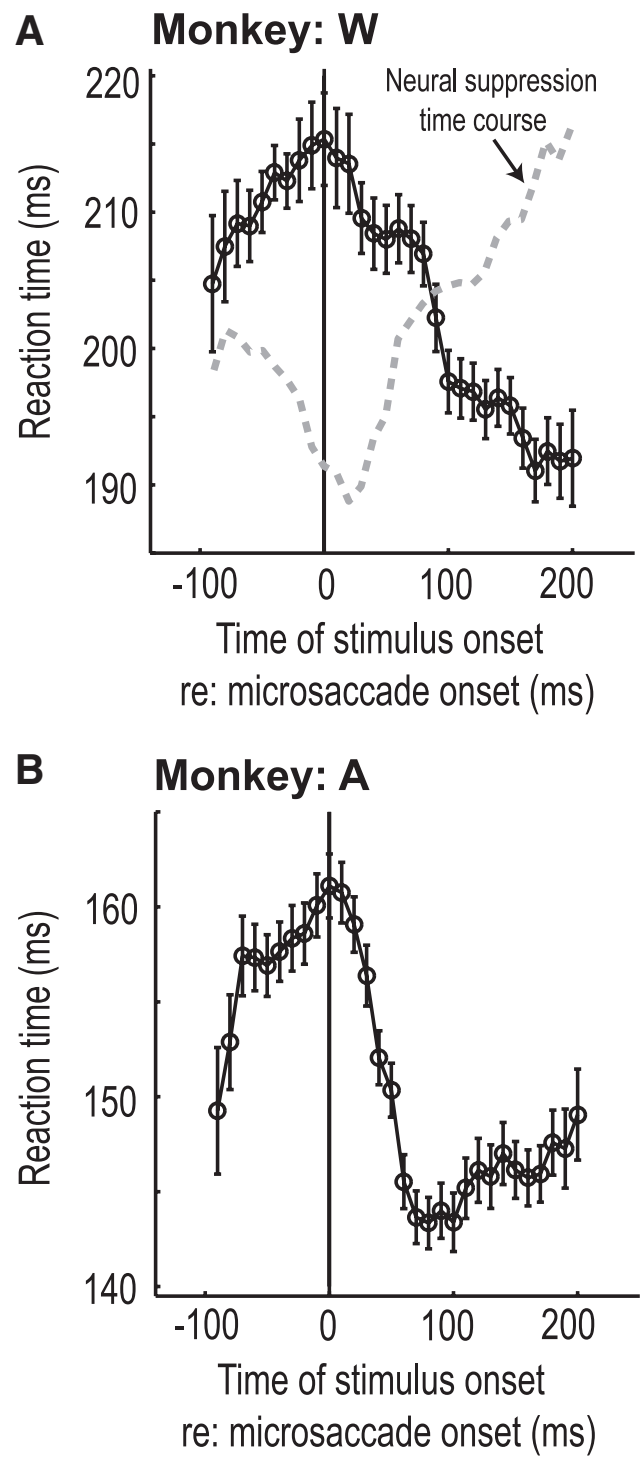

Figure 3. Behavioral correlate of microsaccadic suppression in reaction times. $A, B, \mathrm{RT}$ as a function of stimulus onset relative to microsaccade onset. RTs increased when microsaccade and stimulus onsets coincided. For monkey W $(\boldsymbol{A})$, we also plot the time course of neural suppression effects in the $S C$ (dashed, arbitrary $y$-axis), which shows a similar time course.

ment within $25 \mathrm{~ms}$ of movement onset. Performance recovered to near normal levels only for test stimuli occurring $>50 \mathrm{~ms}$ before or after microsaccades. Similarly, Beeler (1967) found that microsaccadic suppression begins $75-100 \mathrm{~ms}$ before microsaccade onset, reaches maximum effect during the movement, and dissipates by 60-90 ms after the movement. Both results are similar to the neural effects we observed, suggesting that SC visual burst suppression is related to the behavioral correlates of microsaccadic suppression. All of these results are also similar to visual suppression phenomena observed with larger saccades. For example, for saccades $>1-2$ orders of magnitude larger than microsaccades, Diamond et al. (2000) found that suppression precedes movement onset by $75-80 \mathrm{~ms}$ and outlasts movements by $\sim 50 \mathrm{~ms}$. Thus, microsaccadic suppression of SC visual bursts is related to behavioral correlates of both saccadic and microsaccadic suppression of perception.

The time course of microsaccadic suppression of SC visual bursts is similar to the time course of neuronal saccadic (and microsaccadic) suppression observed in other areas. Saccades 
cause suppression of neuronal activity in LGN, starting $\sim 100 \mathrm{~ms}$ before movement onset (Noda and Adey, 1974; Reppas et al., 2002; Sylvester et al., 2005; Royal et al., 2006). Saccades and microsaccades also cause suppression in V1 (Leopold and Logothetis, 1998; Sylvester et al., 2005; Vallines and Greenlee, 2006; Kagan et al., 2008) and extrastriate cortical areas such as V4 (Bosman et al., 2009), MT/MST (Thiele et al., 2002; Ibbotson et al., 2008; Bremmer et al., 2009; Crowder et al., 2009; Herrington et al., 2009), and LIP/VIP (Herrington et al., 2009). In these areas, suppression occurs between approximately -90 and $\sim 50$ ms relative to saccade onset (e.g., Ibbotson et al., 2008), consistent with our observations for microsaccadic suppression of SC bursts. In addition, visual bursts in FEF (Mayo and Sommer, 2008) and superficial SC (Goldberg and Wurtz, 1972; Robinson and Wurtz, 1976; Richmond and Wurtz, 1980) have been studied for stimuli presented after the end of large saccades. In these experiments, visual burst size was reduced by an amount similar to our observations on intermediate-layer SC neurons for microsaccades. It is not known whether FEF or superficial-layer SC neurons also show visual suppression for microsaccades, but it is possible that the suppression we observed is due, at least in part, to properties inherited from these neurons.

The SC is causally involved in microsaccade generation (Hafed et al., 2009), providing an explanation for how microsaccadic suppression of SC visual bursts could occur. SC neurons with foveal RFs exhibit elevated activity around microsaccades (Hafed et al., 2009). The temporal profile of this activity complements the suppression profiles we observed: microsaccaderelated activity increases gradually before movement onset, peaks around movement onset, and gradually decays back to baseline after movement end (Hafed et al., 2009). Thus, microsaccadic suppression of SC visual bursts could occur through inhibition between the neurons contributing to microsaccade generation and the neurons representing the peripheral stimulus. This inhibition is likely mediated by extrinsic pathways to the SC-for example, through an influence of premotor SC activity on LGN (Xue et al., 1994; Thilo et al., 2004).

The finding that microsaccades involve significant suppressive influences on neural activity suggests that these movements, like larger saccades, are associated with active and passive (Wurtz, 2008) processes that ensure perceptual stability in the face of their retinal image shifts. Such processes do not necessarily "shut down" vision completely (Wurtz, 2008), but may modulate neuronal sensitivity to correctly handle potentially misleading visual stimulation caused by microsaccades. It would be interesting to determine whether SC visual burst suppression is the result of changes in the shapes of the RFs, or changes in excitability with no RF changes. Also, even though we observed suppression for microsaccades in all directions, it is possible that important quantitative differences exist for different movement directions relative to RF location. Such differences might be related to the process of perisaccadic RF shifting in SC, LIP, and FEF (Duhamel et al., 1992; Walker et al., 1995; Umeno and Goldberg, 1997).

Finally, the significant suppressive influence of microsaccades is complementary to the fact that these eye movements, along with larger saccades and movements of the head and body, contribute to retinal image motion and therefore can help prevent fading when no other sources of image motion exist. In LGN, V1, V2, V4, and MST, neurons often exhibit enhancement of activity after microsaccades and saccades (Leopold and Logothetis, 1998; Martinez-Conde et al., 2002; Reppas et al., 2002; Royal et al., 2006; Ibbotson et al., 2008; Kagan et al., 2008; Bosman et al., 2009; Bremmer et al., 2009). This postmovement enhancement of sensory activity is likely a result of retinal stimulation caused by eye movements, and is not present in later areas such as LIP (Herrington et al., 2009) that are less dependent on sensory inputs and more closely related to the perceptual evaluations of these inputs (Gottlieb, 2007). Thus, microsaccadic suppression complements microsaccade-induced retinal image motion in its impact on neuronal activity. This is important for perceptual stability, especially for foveal vision and under conditions that make perceptual fading unlikely.

\section{References}

Beeler GW Jr (1967) Visual threshold changes resulting from spontaneous saccadic eye movements. Vision Res 7:769-775.

Boehnke SE, Munoz DP (2008) On the importance of the transient visual response in the superior colliculus. Curr Opin Neurobiol 18:544-551.

Bosman CA, Womelsdorf T, Desimone R, Fries P (2009) A microsaccadic rhythm modulates gamma-band synchronization and behavior. J Neurosci 29:9471-9480.

Bremmer F, Kubischik M, Hoffmann KP, Krekelberg B (2009) Neural dynamics of saccadic suppression. J Neurosci 29:12374-12383.

Crowder NA, Price NSC, Mustari MJ, Ibbotson MR (2009) Direction and contrast tuning of MSTd neurons during saccades. J Neurophysiol 101:3100-3107.

Diamond MR, Ross J, Morrone MC (2000) Extraretinal control of saccadic suppression. J Neurosci 20:3449-3455.

Duhamel JR, Colby CL, Goldberg ME (1992) The updating of the representation of visual space in parietal cortex by intended eye movements. Science 255:90-92.

Fecteau JH, Bell AH, Munoz DP (2004) Neural correlates of the automatic and goal-driven biases in orienting spatial attention. J Neurophysiol 92:1728-1737.

Goldberg ME, Wurtz RH (1972) Activity of superior colliculus in behaving monkey. I. Visual receptive fields of single neurons. J Neurophysiol 35:542-559.

Gottlieb J (2007) From thought to action: the parietal cortex as a bridge between perception, action, and cognition. Neuron 53:9-16.

Hafed ZM, Krauzlis RJ (2008) Goal representations dominate superior colliculus activity during extrafoveal tracking. J Neurosci 28:9426-9439.

Hafed ZM, Goffart L, Krauzlis RJ (2009) A neural mechanism for microsaccade generation in the primate superior colliculus. Science 323:940-943.

Herrington TM, Masse NY, Hachmeh KJ, Smith JE, Assad JA, Cook EP (2009) The effect of microsaccades on the correlation between neural activity and behavior in middle temporal, ventral intraparietal, and lateral intraparietal areas. J Neurosci 29:5793-5805.

Ibbotson MR, Crowder NA, Cloherty SL, Price NSC, Mustari MJ (2008) Saccadic modulation of neural responses: possible roles in saccadic suppression, enhancement, and time compression. J Neurosci 28:1095210960.

Isa T, Hall WC (2009) Exploring the superior colliculus in vitro. J Neurophysiol 102:2581-2593.

Kagan I, Gur M, Snodderly DM (2008) Saccades and drifts differentially modulate neuronal activity in V1: effects of retinal image motion, position, and extraretinal influences. J Vis 8:19.1-19.25.

Kliegl R, Rolfs M, Laubrock J, Engbert R (2009) Microsaccadic modulation of response times in spatial attention tasks. Psychol Res 73:136-146.

Lee PH, Sooksawate T, Yanagawa Y, Isa K, Isa T, Hall WC (2007) Identity of a pathway for saccadic suppression. Proc Natl Acad Sci USA 104:6824-6827.

Leopold DA, Logothetis NK (1998) Microsaccades differentially modulate neural activity in the striate and extrastriate visual cortex. Exp Brain Res 123:341-345.

Martinez-Conde S, Macknik SL, Hubel DH (2002) The function of bursts of spikes during visual fixation in the awake primate lateral geniculate nucleus and primary visual cortex. Proc Natl Acad Sci USA 99:13920-13925.

Martinez-Conde S, Macknik SL, Hubel DH (2004) The role of fixational eye movements in visual perception. Nat Rev Neurosci 5:229-240.

Mayo JP, Sommer MA (2008) Neuronal adaptation caused by sequential visual stimulation in the frontal eye field. J Neurophysiol 100:1923-1935. 
Noda H, Adey WR (1974) Excitability changes in cat lateral geniculate cells during saccadic eye movements. Science 183:543-545.

Reppas JB, Usrey WM, Reid RC (2002) Saccadic eye movements modulate visual responses in the lateral geniculate nucleus. Neuron 35:961-974.

Richmond BJ, Wurtz RH (1980) Vision during saccadic eye movements. II. A corollary discharge to monkey superior colliculus. J Neurophysiol 43:1156-1167.

Robinson DL, Wurtz RH (1976) Use of an extraretinal signal by monkey superior colliculus neurons to distinguish real from self-induced stimulus movement. J Neurophysiol 39:852-870.

Rolfs M, Laubrock J, Kliegl R (2006) Shortening and prolongation of saccade latencies following microsaccades. Exp Brain Res 169:369-376.

Rolfs M, Kliegl R, Engbert R (2008) Toward a model of microsaccade generation: the case of microsaccadic inhibition. J Vis 8:5.1-5.23.

Ross J, Morrone MC, Goldberg ME, Burr DC (2001) Changes in visual perception at the time of saccades. Trends Neurosci 24:113-121.

Royal DW, Sáry G, Schall JD, Casagrande VA (2006) Correlates of motor planning and postsaccadic fixation in the macaque monkey lateral geniculate nucleus. Exp Brain Res 168:62-75.

Sylvester R, Haynes JD, Rees G (2005) Saccades differentially modulate human LGN and V1 responses in the presence and absence of visual stimulation. Curr Biol 15:37-41.

Thiele A, Henning P, Kubischik M, Hoffmann KP (2002) Neural mechanisms of saccadic suppression. Science 295:2460-2462.
Thilo KV, Santoro L, Walsh V, Blakemore C (2004) The site of saccadic suppression. Nat Neurosci 7:13-14.

Umeno MM, Goldberg ME (1997) Spatial processing in the monkey frontal eye field. I. Predictive visual responses. J Neurophysiol 78:1373-1383.

Vallines I, Greenlee MW (2006) Saccadic suppression of retinotopically localized blood oxygen level-dependent responses in human primary visual area V1. J Neurosci 26:5965-5969.

Walker MF, Fitzgibbon EJ, Goldberg ME (1995) Neurons in the monkey superior colliculus predict the visual result of impending saccadic eye movements. J Neurophysiol 73:1988-2003.

Wurtz RH (2008) Neuronal mechanisms of visual stability. Vision Res 48:2070-2089.

Wurtz RH, Goldberg ME (1972) The primate superior colliculus and the shift of visual attention. Invest Ophthalmol 11:441-450.

Wurtz RH, Mohler CW (1976) Organization of monkey superior colliculus: enhanced visual response of superficial layer cells. J Neurophysiol 39:745-765.

Xue JT, Kim CB, Moore RJ, Spear PD (1994) Influence of the superior colliculus on responses of lateral geniculate neurons in the cat. Vis Neurosci 11:1059-1076.

Zuber BL, Stark L (1965) Microsaccades and the velocity-amplitude relationship for saccadic eye movements. Science 150:1459-1460.

Zuber BL, Stark L (1966) Saccadic suppression: elevation of visual threshold associated with saccadic eye movements. Exp Neurol 16:65-79. 